\title{
Patrones de susceptibilidad antimicrobiana de bacterias gramnegativas aisladas de infecciones del tracto urinario en Venezuela: Resultados del estudio SMART 2009-2012
}

\author{
Napoleón Guevara, Manuel Guzmán, Altagracia Merentes, Adele Rizzi, Juana Papaptzikos, \\ Narlesky Rivero, Carmela Oranges, Héctor Villarroel y Yoxsivell Limas
}

\section{Antimicrobial susceptibility patterns of Gram-negative bacteria isolated in urinary tract infections in Venezuela: Results of the SMART study 2009-2012}

Introduction: Antimicrobial resistance of pathogens causing urinary tract infection (UTI) is a growing problem, which complicates their effective treatment. Surveillance is needed to guide appropriate empiric therapy. Aim: to describe the susceptibility patterns of Gram-negative bacteria isolated of patients with UTI to twelve antibiotics as part of the Study for Monitoring Antimicrobial Resistance Trends in Venezuela. Materials and Methods: Between 2009-2012 a total of 472 Gram-negative bacteria were isolated from hospitalized patients with UTI. The isolates were sent to Central Laboratory (Central Laboratory of International Health Management Associates) to confirm their identification, and to make susceptibility testing as recommended by the Clinical and Laboratory Standards Institute. Enterobacteriacea comprised $96.6 \%$ of the total, where Escherichia coli $(76.9 \%)$ and Klebsiella pneumoniae (10.6\%) were the most frequent. Extended-spectrum $\beta$-lactamases (ESBL) was detected in $21.6 \%$ of isolates. Top antimicrobial activity were ertapenem, imipenem, and amikacin $(>90.0 \%)$, slightly lower for amikacin $(85.1 \%)$ in ESBL-producing strains. Resistance rates to fluoroquinolones and ampicillin/sulbactam were high (40 y $64 \%$, respectively). Conclusions: These data suggest a necessary revision of the therapeutic regimens for the empirical treatment of UTI in Venezuela.

Key words: SMART, urinary tract infections, extended-spectrum $\beta$-lactamase, ESBL, susceptibility.

Palabras clave: SMART, infección del tracto urinario, $\beta$-lactamasa de espectro extendido, BLEE, susceptibilidad.

\section{Introducción}

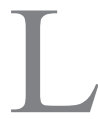

as infecciones del tracto urinario (ITU), son las infecciones más frecuentes en los humanos después de las infecciones respiratorias y gastrointestinales ${ }^{1}$, una causa común de hospitalizaciones y la más frecuente de las infecciones asociadas a cuidados de la salud en hospitales generales en el mundo ${ }^{2,3}$. Se ha considerado como un problema de salud pública, puesto que la incidencia de ITU se estima en 150 millones de casos por años, resultando en un alto costo económico en gastos directos de cuidados en salud ${ }^{4,5}$. La frecuencia es mayor en mujeres que en hombres, estimándose una incidencia de 0,5-0,7 infecciones por año ${ }^{6}$. Además, 25-30\% de las mujeres con ITU tienen episodios recurrentes no relacionados con anormalidad anatómica alguna del tracto urinario. Los factores de riesgo incluyen factores genéticos así como también hábitos de comportamiento ${ }^{5,7,8}$.

Más de 95\% de las ITU están causadas por una única especie bacteriana. Escherichia coli uropatógena causa 70 a $95 \%$ de los episodios de ITU adquirida en la comunidad y $50 \%$ de todos los casos de infecciones nosocomiales ${ }^{5,9,10}$.
Especies menos frecuentes de Enterobacteriaceae, tal como Proteus mirabilis y Klebsiella pneumoniae, y cocáceas grampositivas como Staphylococcus saprophyticus y Streptococcus agalactiae son responsables de la gran mayoría de los episodios restantes. Otras especies gramnegativas y grampositivas son raramente aisladas en ITU no complicadas ${ }^{9,10}$.

En casi todos los casos de ITU, se inicia el tratamiento antimicrobiano empírico antes de tener disponible los resultados del urocultivo, lo que podría incrementar la resistencia de los uropatógenos a los antimicrobianos ${ }^{11}$. Se ha descrito que tanto la cantidad de antimicrobianos utilizado y precisamente cómo son usados contribuye al desarrollo de resistencia ${ }^{12,13}$. Se conoce, entre otras causas, que el uso de antimicrobianos de amplio espectro en lugar de agentes de espectro reducido, favorece la emergencia de la resistencia por una amplia eliminación de la microbiota competitiva susceptible ${ }^{13}$.

Las tasas de resistencia han experimentado importantes variaciones, incrementándose en los últimos 10 años y varían de acuerdo a distintos factores. Por lo tanto, el tratamiento empírico de las ITU requiere la constante
Centro Médico de Caracas, Venezuela (MGB, AM, AR). Hospital de Clínicas Caracas, Venezuela (JP)

Hospital Clínico Universitario de Caracas, Venezuela (NG, NR). MSD Venezuela $(\mathrm{CO}, \mathrm{HV}, \mathrm{YL})$.

Conflictos de interés: Financiamiento: Proyecto Merck Sharp \& Dohme.

Recibido: 16 de diciembre de 2014 Aceptado: 29 de octubre de 2015

Correspondencia a: Napoleón Guevara rnapoleong@gmail.com 
actualización de la susceptibilidad antimicrobiana in vitro de los principales uropatógenos causantes de infección urinaria de la zona, país o institución ${ }^{14} \mathrm{y}$ así, orientar la elección apropiada del tratamiento antimicrobiano empírico. De tal manera que las actividades de vigilancia son necesarias para fundamentar las decisiones terapéuticas, orientar las recomendaciones de políticas y evaluar el impacto de las intervenciones destinadas a contener la resistencia ${ }^{15}$. Tomando en cuenta lo anterior, se desarrolló un programa de monitorización de la resistencia bacteriana, multinacional, denominado Estudio para el Monitoreo de la Tendencia de Resistencia Antimicrobiana, SMART, por sus siglas en inglés (Study for Monitoring Antimicrobial Resistance Trends).

Desde el año 2002, el estudio SMART, fue iniciado por Merck para la vigilancia epidemiológica de la resistencia bacteriana in vitro de los bacilos gramnegativos aerobios $\mathrm{y}$ facultativos a 12 antimicrobianos en infecciones intraabdominales (IIA) en el concierto internacional, en 187 sitios (para el 2013), y desde 2009, fue extendido para incluir la vigilancia de los patógenos gramnegativos que causan ITU. Otro de sus objetivos ha sido identificar cambios en los patrones de susceptibilidad y resistencia en los microorganismos adquiridos en la comunidad $u$ hospitales, incluyendo aquellos que producen $\beta$-lactamasas de espectro extendido (BLEE) ${ }^{16}$. Venezuela forma parte de SMART desde 2002 en cuanto a IIA y desde el año 2009, ha participado en este programa de vigilancia en ITU, por lo que en el presente reporte se documenta los patrones de la susceptibilidad in vitro de bacterias gramnegativas, aisladas de pacientes hospitalizados con ITU, a doce antimicrobianos de uso común, como parte del programa SMART en Venezuela, durante los años 2009-2012.

\section{Materiales y Métodos}

\section{Lugares de estudio}

Desde el último trimestre de 2009 hasta 2012 hubo tres centros en Venezuela que participaron en SMART. Ellos fueron el Hospital Universitario de Caracas, Centro Médico de Caracas y Hospital de Clínicas Caracas; todo localizados en Caracas. El protocolo de SMART contempla la recolección de hasta 50 aislados por año de bacilos gramnegativos provenientes de ITU de cada centro. En 2009, se recolectó en uno de los centros 33 aislados de bacilos gramnegativos de muestras del tracto urinario, y cada uno de los centros participantes recolectó prospectivamente más de 100 muestras durante 2010, 2011 y 2012.

\section{Muestras biológicas}

Se incluyeron aislados de bacilos gramnegativos de pacientes hospitalizados en cada centro con diagnóstico de ITU y que fueron clínicamente significativos $\left(10^{5}\right.$ unidades formadoras de colonias ufc/ml). Se incluyó sólo un aislado por paciente y por especie. Las muestras biológicas fueron identificadas por métodos estándares en los laboratorios de microbiología de los centros participantes. Posteriormente, fueron enviadas al Laboratorio Central de Referencia (Central Laboratory of International Health Management Associates IHMA, Inc., Schaumburg, IL, USA) para las pruebas de susceptibilidad y confirmar la identificación. Los aislados obtenidos de pacientes con menos de $48 \mathrm{~h}$ de hospitalización se clasificaron como adquiridos en la comunidad y aquellos procedentes de pacientes con 48 h o más de hospitalización se clasificaron como nosocomiales.

\section{Pruebas de susceptibilidad antimicrobiana}

Los antimicrobianos evaluados fueron amikacina (AMK), ampicilina/sulbactam (A/S), cefepime (CPE), cefotaxima (CFT), cefoxitin (CFX), ceftazidima (CAZ), ceftriaxona (CAX), ciprofloxacina (CP), ertapenem (ETP), imipenem (IMP), levofloxacina (LVX), y piperacilina/tazobactam $(\mathrm{P} / \mathrm{T})$. Para determinar la concentración inhibitoria mínima (CIM) de los 12 antimicrobianos, se utilizó el método de microdilución en caldo con paneles deshidratados MicroScan ${ }^{\circledR}$ (Siemens), de acuerdo a las recomendaciones del Instituto de Estándares Clínicos y de Laboratorio (Clinical and Laboratory Standards Institute, CLSI). Los criterios de interpretación de las CIM se rigieron por los establecidos por $\mathrm{CLSI}^{17}$.

\section{Confirmación de B-lactamasas de espectro extendido}

Se realizó la prueba confirmatoria para la detección fenotípica de la producción de BLEE. Los aislados de $P$. mirabilis, $K$. pneumoniae, $K$. oxytoca y $E$. coli se clasificaron como productores de BLEE cuando hubo reducción de al menos ocho veces las CIM para ceftazidima y/o cefotaxima evaluadas en combinación con ácido clavulánico comparados con las CIM del antimicrobiano cuando fue evaluado solo ${ }^{17}$.

\section{Análisis estadístico}

Para el análisis estadístico se utilizó el programa Stata 12.1 (StataCorp LP). El nivel de significancia establecido fue de $5 \%(\alpha=0,05)$. Para verificar la normalidad de las variables numéricas se utilizó la prueba de Shapiro Wilk ${ }^{18}$. Se utilizaron medidas de resumen para la descripción de las variables. Las tasas de susceptibilidad in vitro se calcularon con sus respectivos intervalos del confianza al 95\% (IC95\%). En el caso de variables sin distribución normal, se usó la prueba de la U de Mann Whitney o el ANOVA de un tratamiento de Kruskal-Wallis cuando fue apropiado, para comparar diferencias en la distribución de una variable cuantitativa según categorías de una variable cualitativa ${ }^{19}$. Cuando las variables cuantitativas tuvieron distribución normal, se usó la prueba $t$ de Student 
o ANOVA de un tratamiento según número de categorías de la variable cualitativa ${ }^{20}$. Para comparar diferencias en proporciones, se usó la prueba de Chi cuadrado $\left(\chi^{2}\right)$ o la prueba exacta de Fisher a dos extremos cuando fue adecuado $^{19}$.

\section{Resultados}

De 2009 a 2012, se incluyó un total de 472 aislados de bacilos gramnegativos en los tres centros participantes: Hospital Universitario de Caracas (n: 148; 31,4\%), Centro Médico de Caracas (n: 173; 36,7\%) y Hospital de Clínicas Caracas (n: 151; 32,0). Las unidades o servicios más frecuentes de donde procedieron los pacientes en estos centros fueron: Sala de Emergencia (n: 189; 40,0\%), Medicina General (n: 123; 26,1\%), Cirugía General (n: 44; 9,3\%) y Pediatría General (n: 43; 9,1\%). Más de la mitad de las infecciones (n: 250; 53,0\%) fueron adquiridas en la comunidad, $38,6 \%(182 / 472)$ fueron clasificadas como nosocomiales y hubo $8,5 \%$ (40/472) que no fueron especificadas al momento de la toma de muestra. Ninguno de los aislados obtenidos en el año 2009 (n: 33; 7,0\%) fue clasificado según el lugar donde fue adquirida la infección. En los centros participantes fueron más frecuentes las infecciones adquiridas en la comunidad (mayor a 78\%) excepto para el Centro Médico de Caracas (23/148; $15,5 \%)$, esta diferencia fue estadísticamente significativa $(\mathrm{p}<0,00001)$.

La mitad de los pacientes de donde se obtuvieron los aislados tenían edad de 50 años o más y un cuarto del total $(25,0 \%)$ tuvieron edades superiores a 70 años. La mayoría fueron mujeres $(72,7 \%)$, en edades comprendidas entre 1 y 99 años. La composición por edad entre ambos sexos fue significativamente distinta (p: 0,0428), observándose la mediana de edad de los hombres $(57,5$; IQR: 46,5) mayor que la de las mujeres (46; IQR: 42). Igualmente, la composición por grupos de edad de estos pacientes fue significativamente distinta según el origen de la fuente de infección, observándose grupos de mayor edad en las infecciones nosocomiales (Mediana: 58,5; IQR: 47) comparado con las infecciones adquiridas en la comunidad (Mediana: 43; IQR: 41) $(\mathrm{p}=0,0064)$.

Las especies pertenecientes a la familia Enterobacteriaceae comprendieron $96,6 \%(456 / 472)$ de todos los aislados. Escherichia coli $(363 ; 76,9 \%)$, K. pneumoniae (50; $10,6 \%)$ y $P$. mirabilis $(20 ; 4,2 \%)$ fueron las especies de Enterobacteriaceae más frecuentes. Las únicas especies aisladas no pertenecientes a la familia Enterobacteriaceae $(3,4 \% ; 16 / 472)$ fueron Pseudomonas aeruginosa (14/16) y Acinetobacter baumannii (2/16). Los aislados procedieron predominantemente de orina (n: $450 ; 95,3 \%$ ), vejiga urinaria (n: $8 ; 1,7 \%$ ) y riñones (n: $8 ; 1,7 \%$ ). Solamente $E$. coli $(16 / 22 ; 72,7 \%)$, Enterobacter cloacae (1/22; 4,6\%), Enterobacter aerogenes $(1 / 22 ; 4,6 \%)$, K. pneumoniae
$(2 / 22 ; 9,1 \%)$ y $K$. oxytoca $(2 / 22 ; 9,1 \%)$ se aislaron de ubicaciones diferentes a la orina.

Las tasas de susceptibilidad de los patógenos gramnegativos causantes de ITU a los antimicrobianos se presentan en la Tabla 1. El antimicrobiano con mayor actividad in vitro fue ertapenem (95,3\%; IC95\%: 93,097,1), seguido por amikacina (93,8\%; IC95\%:91,3-95,8), imipenem (93,2\%; IC95\%: 90,6-95,3), piperacilina/tazobactam $(85,0 \%$; IC95\%: $81,4-88,1)$ y cefoxitina $(84,5 \%$; IC95\%: 81,0-87,8). El agente antimicrobiano con la tasa de susceptibilidad más baja fue ampicilina/sulbactam (36,0\%; IC95\%: 31,8-40,5), seguido de ciprofloxacina $(58,38 \%$; IC95\%: 53,7-62,8) y levofloxacina $(60,0 \%$; IC95\%: 55,4-64,4). Las tasas de susceptibilidad de los otros agentes $\beta$-lactámicos fluctuaron entre 72,9 y $81,8 \%$.

En general, las especies provenientes de la comunidad fueron más sensibles a todos los antimicrobianos evaluados (40,0-97,6\%) comparado con aquellas adquiridas en los establecimientos de salud (31,3-94,0\%) (Tabla 2), siendo estas diferencias estadísticamente significativas para todos los antimicrobianos, excepto para ampicilina/ sulbactam (p: 0,064), cefoxitina (p: 0,098), imipenem (p: 0,570) y piperacilina/tazobactam (p: 0,543) (Tabla 2). Para ertapenem, amikacina e imipenem, las tasas de susceptibilidad fueron superiores a 90,0\% en ambos grupos. Los aislados de origen comunitario fueron más sensibles a ertapenem (97,6\%), seguido de amikacina $(96,8 \%)$ e imipenem $(95,2 \%)$; teniendo las menores tasas de susceptibilidad a ampicilina/sulbactam (40,0\%) y ciprofloxacina $(64,0 \%)$. Situación similar se observó para las especies de origen nosocomial.

Acinetobacter baumannii no fue susceptible a los antimicrobianos evaluados. Para $P$. aeruginosa, las tasas de susceptibilidad variaron entre 7,1 y $57,1 \%$, con las tasas más altas para amikacina, ceftazidima, imipenem y piperacilina/tazobactam, cada una con $57,1 \%$.

Se analizó la producción de BLEE en 436 aislados pertenecientes a Enterobacteriaceae, confirmándose positividad en 21,6\% (94/436). Fueron productoras de BLEE, $21,8 \%(79 / 363)$ de las especies de $E$. coli y $24,0 \%(12 / 50)$ de K. pneumoniae. La proporción de especies productoras de BLEE en las infecciones adquiridas en la comunidad $(37 ; 15,6 \%)$ fue menor que la proporción de cepas productoras de BLEE en las infecciones nosocomiales (n: 47; $28,7 \%$ ), siendo esta diferencia estadísticamente significativa (p: 0,001). En la Figura 1 se presenta la frecuencia de las especies evaluadas para producción de BLEE de acuerdo a su procedencia comunitaria o nosocomial. En $10,6 \%$ (10/94) de las especies productoras de BLEE no se determinó el origen donde se adquirió la infección.

En $89,9 \%$ (71/79) de las especies de E. coli y $83,3 \%$ (10/12) de K. pneumoniae productoras de BLEE se determinó el origen de la infección, observándose en ambas mayor proporción en las infecciones nosocomiales $(56,3$ 
Tabla 1. Susceptibilidad (\%) in vitro de las especies aisladas causantes de infecciones del tracto urinario en Venezuela, 2009-2012

\begin{tabular}{|c|c|c|c|c|c|c|c|c|c|c|c|c|c|c|}
\hline \multirow[t]{2}{*}{ Bacteria } & \multirow{2}{*}{\multicolumn{2}{|c|}{$\begin{array}{c}\text { n de aislados } \\
\text { (\%) }\end{array}$}} & \multicolumn{12}{|c|}{ Agente antimicrobiano } \\
\hline & & & ETP & AMK & $A / S$ & CPE & CFT & CFX & CAZ & CAX & $\mathrm{CP}$ & IMP & LVX & PT \\
\hline Total & 472 & $(100,0)$ & 95,3 & 93,9 & 36,0 & 81,8 & 72,9 & 84,5 & 78,0 & 72,9 & 58,3 & 93,2 & 60,0 & 85,0 \\
\hline Enterobacteriaceae & 456 & $(96,6)$ & 98,5 & 95,4 & 37,1 & 83,1 & 75,2 & 87,3 & 79,0 & 75,2 & 59,0 & 94,7 & 60,8 & 86,2 \\
\hline Escherichia coli & 363 & $(76,9)^{\delta}$ & 99,5 & 97,0 & 33,6 & 82,9 & 76,9 & 83,5 & 80,7 & 76,9 & 55,4 & 99,7 & 57,0 & 88,2 \\
\hline E. coli $(\mathrm{BLEE}+)$ & 79 & $(21,8)^{\phi}$ & 100,0 & 89,9 & 8,9 & 24,1 & 2,5 & 78,5 & 19,0 & 2,5 & 2,5 & 100,0 & 8,9 & 73,4 \\
\hline E. coli (BLEE-) & 283 & $(78,2)^{\phi}$ & 99,7 & 98,9 & 40,6 & 99,7 & 97,9 & 92,9 & 98,2 & 97,9 & 70,3 & 99,7 & 70,7 & 92,6 \\
\hline Klebsiella pneumoniae & 50 & $(10,6)^{\delta}$ & 94,0 & 86,0 & 52,0 & 80,0 & 70,0 & 90,0 & 74,0 & 70,0 & 70,0 & 94,0 & 70,0 & 76,0 \\
\hline K. pneumoniae (BLEE + ) & 12 & $(24,0)^{\phi}$ & 91,7 & 66,7 & 0,0 & 41,7 & 16,7 & 91,7 & 25,0 & 16,7 & 41,7 & 91,7 & 41,7 & 41,7 \\
\hline K. pneumoniae (BLEE-) & 38 & $(76,0)^{\phi}$ & 94,7 & 92,1 & 68,4 & 92,1 & 86,8 & 89,5 & 89,5 & 86,8 & 79,0 & 94,7 & 79,0 & 86,8 \\
\hline Proteus mirabilis & 20 & $(4,2)^{\delta}$ & 100,0 & 100,0 & 80,0 & 95,0 & 90,0 & 95,0 & 80,0 & 90,0 & 85,0 & 30,0 & 85,0 & 95,0 \\
\hline P. mirabilis (BLEE + ) & 1 & $(5,0)^{\phi}$ & 100,0 & 100,0 & 0,0 & 100,0 & 0,0 & 100,0 & 0,0 & 0,0 & 0,0 & 100,0 & 0,0 & 100,0 \\
\hline P. mirabilis (BLEE-) & 19 & $(95,0)^{\phi}$ & 100,0 & 100,0 & 84,2 & 94,7 & 94,7 & 94,7 & 84,2 & 94,7 & 89,5 & 26,3 & 89,5 & 94,7 \\
\hline Enterobacter cloacae & 5 & $(1,1)$ & 60,0 & 80,0 & 20,0 & 80,0 & 20,0 & 0,0 & 20,0 & 20,0 & 80,0 & 80,0 & 80,0 & 40,0 \\
\hline Enterobacter aerogenes & 5 & $(1,1)$ & 100,0 & 100,0 & 20,0 & 100,0 & 40,0 & 0,0 & 60,0 & 40,0 & 100,0 & 60,0 & 100,0 & 60,0 \\
\hline Klebsiella oxytoca & 4 & $(0,9)^{8}$ & 100,0 & 50,0 & 0,0 & 50,0 & 50,0 & 100,0 & 50,0 & 50,0 & 50,0 & 100,0 & 50,0 & 50,0 \\
\hline K. oxytoca (BLEE + ) & 2 & $(50,0)^{\phi}$ & 100,0 & 0,0 & 0,0 & 0,0 & 0,0 & 100,0 & 0,0 & 0,0 & 0,0 & 100,0 & 0,0 & 0,0 \\
\hline K. oxytoca (BLEE-) & 2 & $(50,0)^{\phi}$ & 100,0 & 100,0 & 0,0 & 100,0 & 100,0 & 100,0 & 100,0 & 100,0 & 100,0 & 100,0 & 100,0 & 100,0 \\
\hline Citrobacter freundii & 3 & $(0,6)$ & 100,0 & 100,0 & 66,7 & 66,7 & 66,7 & 0,0 & 66,7 & 66,7 & 33,3 & 100,0 & 66,7 & 100,0 \\
\hline Morganella morganii & 2 & $(0,4)$ & 100,0 & 100,0 & 0,0 & 100,0 & 100,0 & 100,0 & 100,0 & 100,0 & 50,0 & 50,0 & 50,0 & 100,0 \\
\hline Proteus vulgaris & 1 & $(0,2)$ & 100,0 & 100,0 & 0,0 & 100,0 & 0,0 & 100,0 & 100,0 & 0,0 & 100,0 & 0,0 & 100,0 & 100,0 \\
\hline Proteus penneri & 1 & $(0,2)$ & 100,0 & 100,0 & 0,0 & 100,0 & 0,0 & 100,0 & 100,0 & 0,0 & 100,0 & 0,0 & 100,0 & 100,0 \\
\hline Salmonella, non-speciated & 1 & $(0,2)$ & 100,0 & 100,0 & 100,0 & 100,0 & 100,0 & 100,0 & 100,0 & 100,0 & 0,0 & 100,0 & 100,0 & 100,0 \\
\hline Serratia marcescens & 1 & $(0,2)$ & 100,0 & 100,0 & 0,0 & 100,0 & 100,0 & 0,0 & 100,0 & 100,0 & 100,0 & 100,0 & 100,0 & 100,0 \\
\hline No-Enterobacteriaceae & 16 & $(3,4)$ & 6,3 & 50,0 & 6,3 & 43,8 & 6,3 & 6,3 & 50,0 & 6,3 & 37,5 & 50,0 & 37,5 & 50,0 \\
\hline Acinetobacter baumannii & 2 & $(0,4)$ & 0,0 & 0,0 & 0,0 & 0,0 & 0,0 & 0,0 & 0,0 & 0,0 & 0,0 & 0,0 & 0,0 & 0,0 \\
\hline Pseudomonas aeruginosa & 14 & $(3,0)$ & 7,1 & 57,1 & 7,1 & 50,0 & 7,1 & 7,1 & 57,1 & 7,1 & 42,9 & 57,1 & 42,9 & 57,1 \\
\hline
\end{tabular}

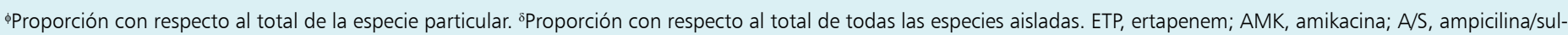
bactam; CPE, cefepime; CFT, cefotaxima; CFX, cefoxitina; CAZ, ceftazidima; CAX, ceftriaxona; CP, ciprofloxacina; IMP, imipenem; LVX, levofloxacina; P/T, piperacilina/tazobactam.

Tabla 2. Susceptibilidad (\%) in vitro de las especies aisladas causantes de infecciones del tracto urinario según procedencia de la infección, Venezuela, 2009-2012

\begin{tabular}{|c|c|c|c|c|c|c|c|}
\hline \multirow{3}{*}{$\begin{array}{c}\text { Agente } \\
\text { antimicrobiano }\end{array}$} & \multirow[t]{3}{*}{ Total } & \multirow[t]{3}{*}{ IC $95 \%$} & \multicolumn{4}{|c|}{ Origen de la infección } & \multirow{3}{*}{$\begin{array}{c}\text { Valor } \\
\mathbf{p}^{\delta}\end{array}$} \\
\hline & & & \multicolumn{2}{|c|}{ Comunidad } & \multicolumn{2}{|c|}{ Nosocomial } & \\
\hline & & & Total & IC 95\% & Total & IC $95 \%$ & \\
\hline ETP & 95,3 & $93,0-97,1$ & 97,6 & $94,8-99,1$ & 93,4 & $88,8-96,5$ & 0,031 \\
\hline AMK & 93,9 & $91,3-95,4$ & 96,8 & $93,8-98,6$ & 91,2 & $86,1-94,9$ & 0,012 \\
\hline A/S & 36,0 & $31,7-40,5$ & 40,0 & $33,9-46,4$ & 31,3 & $24,7-38,6$ & 0,064 \\
\hline CPE & 81,8 & $78,0-85,2$ & 87,2 & $82,4-91,1$ & 76,9 & $70,1-82,8$ & 0,005 \\
\hline CFT & 72,9 & $68,6-76,8$ & 80,4 & $74,9-85,1$ & 66,5 & $59,1-73,3$ & 0,001 \\
\hline CAZ & 84,5 & $74,0-81,6$ & 84,4 & $79,3-88,7$ & 72,5 & $65,4-78,9$ & 0,003 \\
\hline CFX & 78,0 & $81,0-87,7$ & 87,6 & $82,9-91,4$ & 81,9 & $75,5-87,2$ & 0,098 \\
\hline CAX & 72,9 & $68,6-76,8$ & 80,4 & $74,9-85,1$ & 66,5 & $59,1-73,3$ & 0,001 \\
\hline $\mathrm{CP}$ & 58,3 & $53,7-62,8$ & 64,0 & $57,7-70,0$ & 53,8 & $46,3-61,2$ & 0,034 \\
\hline IMP & 93,2 & $90,6-95,3$ & 95,2 & $91,8-97,5$ & 94,0 & $89,4-96,9$ & 0,570 \\
\hline LVX & 60,0 & $55,4-64,4$ & 66,8 & $60,6-72,6$ & 54,4 & $46,9-61,8$ & 0,009 \\
\hline $\mathrm{P} / \mathrm{T}$ & 85,0 & $81,4-88,1$ & 87,2 & $82,4-91,1$ & 85,2 & $79,2-90,0$ & 0,543 \\
\hline
\end{tabular}

${ }^{\delta}$ Prueba de $\chi^{2}$. ETP, ertapenem; AMK, amikacina; AVS, ampicilina/sulbactam; CPE, cefepime; CFT, cefotaxima; CFX, cefoxitina; CAZ, ceftazidima; CAX, ceftriaxona; CP, ciprofloxacina; IMP, imipenem; LVX, levofloxacina; $\mathrm{P} / \mathrm{T}$, piperacilina/tazobactam. y $60,0 \%$, respectivamente). Se observó una tendencia decreciente estadísticamente significativa (p: 0,023) en la proporción de especies productoras de BLEE en las infecciones nosocomiales para los años estudiados (2010: 36,5\%; 2011: $29,4 \%$ y 2012: $21,3 \%$ ), no observándose tendencia clara para estas especies de origen comunitario (p: 0,395 ) ni considerando el total de especies productoras de BLEE independientemente del origen de la infección (p: 0,805). Las tasas de susceptibilidad in vitro para cada antimicrobiano evaluado según la producción de BLEE se muestran en la Tabla 1.

La susceptibilidad en las especies productoras de BLEE fueron mayores para ertapenem e imipenem ( $98,9 \%$ cada uno), amikacina $(85,1 \%)$, cefoxitin $(80,9 \%)$ y piperacilina/tazobactam (68,1\%). Para el resto de los antimicrobianos, las tasas de susceptibilidad no sobrepasaron el 27,0\% (4,4-26,6\%). Las especies no productoras de BLEE tuvieron tasas de susceptibilidad sobre $90,0 \%$ para todos los antimicrobianos, excepto para ampicilina/ sulbactam (45,9\%), ciprofloxacina y levofloxacina $(72,5$ y $72,8 \%$, respectivamente). Hubo tasas de susceptibilidad similares, tanto productores o no de BLEE, para E. coli 
con ertapenem, imipenem y amikacina (mayores a 89\%) (Figura 2). Para $K$. pneumoniae, hubo tasas de susceptibilidad similares para ertapenem, imipenem y cefoxitina, todas sobre $89,0 \%$. En el caso de P. mirabilis, en general se observó una elevada resistencia frente a imipenem $(70,0 \%)$ a expensas de los aislados no productores de BLEE (73,7\%).

Para todos los antimicrobianos evaluados, las tasas de susceptibilidad de $E$. coli fueron mayores en los aislados procedentes de infecciones adquiridas en la comunidad que en las procedentes de infecciones nosocomiales (Tabla 3). La resistencia a ciprofloxacina fue elevada en cepas de E. coli productoras de BLEE (más de 90\%) y algo menor en cepas no productoras de BLEE (mayor de $25 \%$ ), siendo esto similar para levofloxacina. La resistencia a fluoroquinolonas fue ligeramente menor en cepas de origen comunitario que en cepas nosocomiales. Por otro lado, las tasas de susceptibilidad a los carbapenémicos fueron superiores a 99,0\%, indistintamente de su origen comunitario o nosocomial. Para los aislados productores de BLEE, la resistencia a ampicilina/sulbactam fue mayor del $60,0 \%$ en los aislados comunitarios y por encima de $90 \%$ en los aislados nosocomiales.

En ertapenem e imipenem, hubo porcentajes consistentemente elevados de susceptibilidad (mayores a 97,0\%) frente a las especies productoras de BLEE en todos los años de estudio. Se observó ligero incremento de la susceptibilidad para cefoxitina y ceftazidima con los años; sin embargo, no fue estadísticamente significativo. Los únicos antimicrobianos que tuvieron una tendencia franca hacia la disminución de la susceptibilidad para los aislados productores de BLEE desde el año 2010 fue para ciprofloxacina y levofloxacina pero, al igual que con el resto de los antimicrobianos, no hubo evidencias que este cambio fuese estadísticamente significativo.

Se observó una tendencia muy discreta, no significativa, de incremento de la susceptibilidad general para ertapenem, amikacina, cefepime, ceftazidima, ciprofloxacina y levofloxacina desde el año 2009 al 2012 (Figura 3). Sin embargo, para Enterobacteriacea se observaron tasas de susceptibilidad consistentemente elevadas a ertapenem y amikacina, en ambos por encima de 93,0\% durante todos los años. El tercer antimicrobiano con mayores tasas de actividad in vitro durante el período estudiado fue imipenem, por encima de 90,0\%, excepto para el año 2009, cuando presentó tasa de $82,1 \%$. En el caso de piperacilina/tazobactam, las tasas de actividad in vitro tuvieron un rango entre 82,1 y $91,3 \%$. Los valores más bajos se observaron con ampicilina/sulbactam, con tasas inferiores a $41,0 \%$ durante todo los años.

\section{Discusión}

Las ITU son una importante causa de solicitud de atención médica en todo el mundo. En Venezuela, ocupó el sexto y séptimo lugar como causa más frecuente de consultas en los centros de salud en los años 2009 y 2010, y se situó entre las primeras 15 causas de consulta para el año $2011^{21-23}$. Es una patología muy frecuente en la comunidad y de las infecciones nosocomiales son las más comunes ${ }^{24}$. Su gravedad varía según su asociación con otros estados mórbidos, malformaciones anatómicas y procedimientos invasores de vías urinarias, entre otros.

En concordancia a lo tradicionalmente descrito para las ITU, la mayoría de los pacientes incluidos en este estudio fueron del sexo femenino en todos los grupos etarios $(72,7 \%)$, con similar proporción tanto en

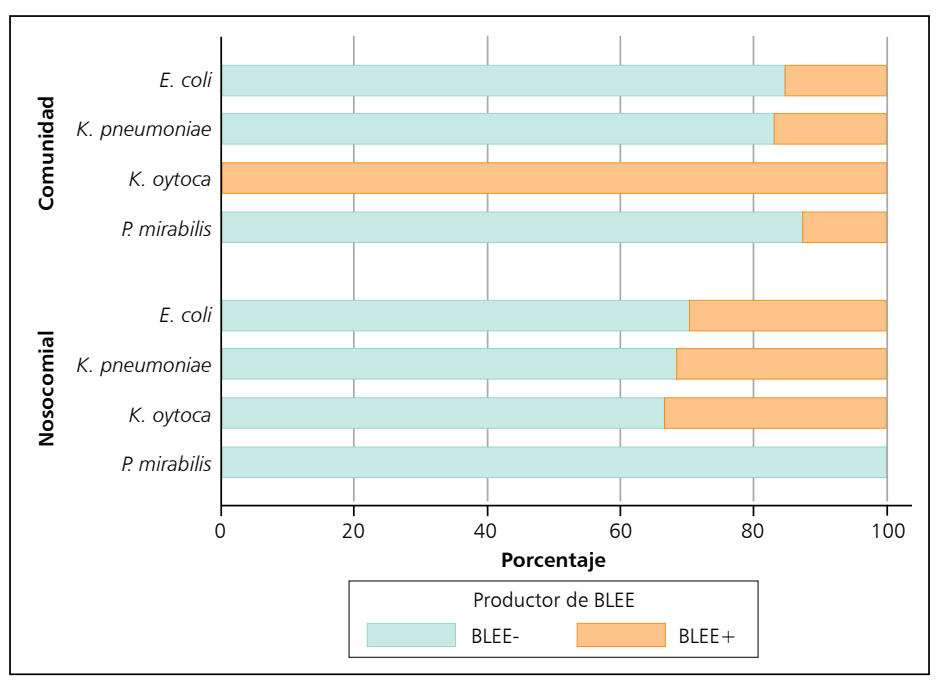

Figura 1. Especies de bacterias gramnegativas aisladas de pacientes con infección del tracto urinario según producción de BLEE y origen de la infección, Venezuela 2009-2012.

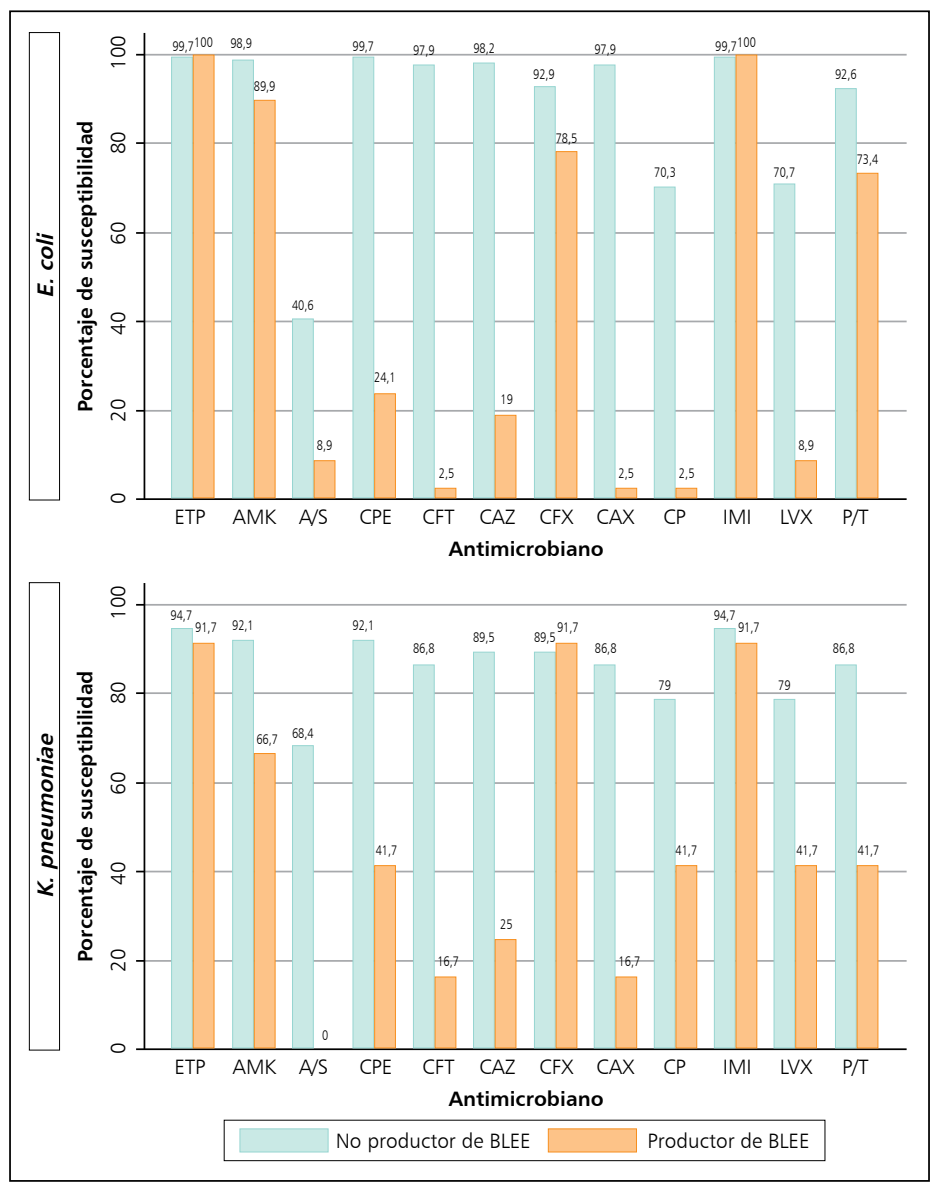

Figura 2. Susceptibilidad in vitro de Escherichia coli y Klebsiella pneumoniae a los antimicrobianos según producción de BLEE, Venezuela 2009-2012. ETP, ertapenem; AMK, amikacina; AVS, ampicilina/sulbactam; CPE, cefepime; CFT, cefotaxima; CFX, cefoxitin; CAZ, ceftazidima; CAX, ceftriaxona; CP, ciprofloxacina; IMP, imipenem; LVX, levofloxacina; P/T, piperacilina/tazobactam. 
Tabla 3. Susceptibilidad (\%) in vitro de Escherichia coli aislada de infecciones del tracto urinario según procedencia de la infección y producción de BLEE, Venezuela 2009-2012

\begin{tabular}{|c|c|c|c|c|c|c|c|c|c|c|}
\hline \multirow{3}{*}{$\begin{array}{l}\text { Agente } \\
\text { antimicrobiano }\end{array}$} & \multicolumn{10}{|c|}{ Origen de la infección } \\
\hline & \multicolumn{4}{|c|}{ General } & \multicolumn{3}{|c|}{ Comunidad } & \multicolumn{3}{|c|}{ Nosocomial } \\
\hline & BLEE- & BLEE+ & Total & IC $95 \%$ & BLEE- & BLEE+ & Total & BLEE- & BLEE+ & Total \\
\hline ETP & 99,6 & 100,0 & 99,7 & $98,5-100,0$ & 99,4 & 100,0 & 99,5 & 100,0 & 100,0 & 99,3 \\
\hline AMK & 98,9 & 89,9 & 97,0 & $94,6-98,5$ & 100,0 & 90,3 & 98,5 & 97,9 & 90,0 & 95,6 \\
\hline A/S & 40,6 & 8,9 & 33,7 & $28,8-38,8$ & 43,1 & 12,9 & 38,5 & 38,5 & 5,0 & 28,5 \\
\hline CPE & 99,6 & 24,1 & 83,1 & $78,9-86,9$ & 99,4 & 25,8 & 88,3 & 100,0 & 22,5 & 76,6 \\
\hline CFT & 97,9 & 2,5 & 77,1 & $72,4-81,3$ & 99,4 & 0,0 & 84,4 & 97,9 & 5,0 & 70,1 \\
\hline CAZ & 98,2 & 19,0 & 80,9 & $76,5-84,9$ & 99,4 & 22,6 & 87,8 & 97,9 & 20,0 & 74,5 \\
\hline CFX & 92,9 & 78,5 & 89,8 & $86,2-92,7$ & 94,3 & 74,2 & 91,2 & 92,7 & 82,5 & 89,1 \\
\hline CAX & 97,9 & 2,5 & 77,1 & $72,4-81,3$ & 99,4 & 0,0 & 84,4 & 97,9 & 5,0 & 70,1 \\
\hline $\mathrm{CP}$ & 70,3 & 2,5 & 55,5 & $50,2-60,7$ & 72,4 & 3,2 & 62,0 & 69,8 & 2,5 & 49,6 \\
\hline IMP & 99,6 & 100,0 & 99,7 & $98,5-100,0$ & 99,4 & 100,0 & 99,5 & 100,0 & 100,0 & 100,0 \\
\hline LVX & 70,7 & 8,9 & 57,2 & $51,9-62,3$ & 73,0 & 16,1 & 64,4 & 69,8 & 5,0 & 50,4 \\
\hline $\mathrm{P} / \mathrm{T}$ & 92,6 & 73,4 & 88,4 & $84,6-91,5$ & 92,5 & 71,0 & 89,3 & 93,6 & 80,0 & 89,1 \\
\hline
\end{tabular}

ETP, ertapenem; AMK, amikacina; A/S, ampicilina/sulbactam; CPE, cefepime; CFT, cefotaxima; CFX, cefoxitina; CAZ, ceftazidima; CAX, ceftriaxona; CP, ciprofloxacina; IMP, imipenem; LVX, levofloxacina; P/T, piperacilin/tazobactam.
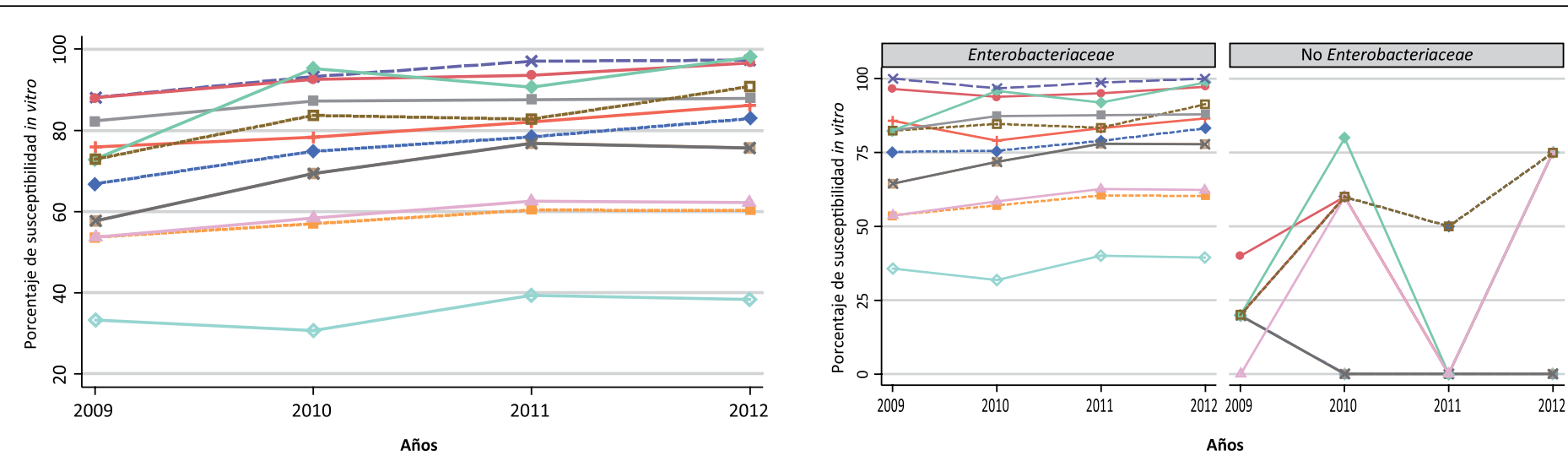

Años

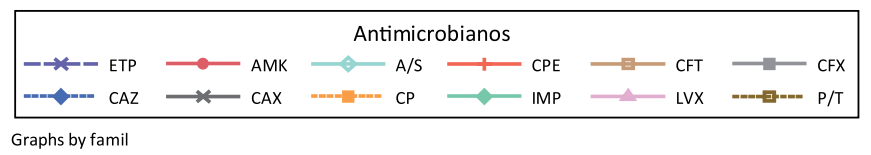

Figura 3. Susceptibilidad in vitro anual de los bacilos gramnegativos aislados de infecciones del tracto urinario a los antimicrobianos estudiados según familia bacteriana, Venezuela 2009-2012. ETP, ertapenem; AMK, amikacina; A/S, ampicilina/sulbactam; CPE, cefepime; CFT, cefotaxima; CFX, cefoxitin; CAZ, ceftazidima; CAX, ceftriaxona; CP, ciprofloxacina; IMP, imipenem; LVX, levofloxacina; P/T, piperacilina/tazobactam.

infecciones de origen nosocomial como en las adquiridas en la comunidad. Esta mayor frecuencia en mujeres y los factores de riesgo asociados con la recurrencia de infección en este grupo ha sido ampliamente estudiado ${ }^{6-8}$.

En forma similar a lo reportado previamente en otros países $^{25,26}$ y en Venezuela ${ }^{27-29}$, en ITU complicadas y no complicadas $^{6,30}$, la especie más frecuentemente aislada tanto de pacientes con infecciones adquiridas en la comunidad como de infecciones nosocomiales fue $E$. coli (76,9\%), seguida de otros bacilos entéricos gramnegativos como $K$. pneumoniae $(10,6 \%)$, P. mirabilis $(4,2 \%)$ y no entéricos como $P$. aeruginosa $(3,0 \%)$. 
Las infecciones causadas por bacterias gramnegativas tienen características de especial preocupación, puesto que son altamente eficientes en la regulación o adquisición de genes que codifican para resistencia a antimicrobianos, especialmente en la presencia de presión de selección con antimicrobianos ${ }^{24}$. Estos mecanismos son diversos; sin embargo, la producción de BLEE es el factor contribuyente más importante de resistencia a $\beta$-lactámicos en bacterias gramnegativas, más frecuentemente reportadas en $K$. pneumoniae, $K$. oxytoca y $E$. coli, pero también reportadas en Citrobacter spp, Enterobacter spp, Proteus spp, Salmonella spp, Serratia spp y otros géneros de enterobacterias, además de microorganismos no entéricos como $A$. baumannii y $P$. aeruginosa ${ }^{31}$. La distribución de estas cepas es heterogénea y la prevalencia de las BLEE se ha incrementado progresivamente en el mundo ${ }^{32}$.

En este sentido, los datos obtenidos muestran que la proporción de aislados productores de BLEE fueron superiores a los descritos para centros de atención médica en Caracas $(16,8 \%)^{33,34}$. Sin embargo, específicamente para E. coli y K. pneumoniae, la proporción de aislados productores de BLEE fue inferior a lo reportado en centros hospitalarios de otras regiones del país $(39,0$ y $52,5 \%$, respectivamente) $)^{35}$. Esto podría deberse en parte a que los aislados sólo procedían de pacientes en cuidados intensivos, además que no fueron exclusivos de muestras procedentes del tracto urinario. Asimismo, la proporción de cepas productoras de BLEE para estas dos especies bacterianas en el presente estudio fue similar, de $29,4 \%$ para $E$. coli y $31,5 \%$ para $K$. pneumoniae en las infecciones nosocomiales y de $15,1 \%$ para $E$. coli y $16,7 \%$ para $K$. pneumoniae en las infecciones que procedían de la comunidad. Además, se observó una tendencia decreciente en la proporción de cepas productoras de BLEE en las infecciones nosocomiales en el lapso estudiado de $36,5 \%$ en 2010 a $21,3 \%$ en 2012 , a expensas básicamente de E. coli.

Por otro lado, la proporción de bacterias productoras de BLEE en las infecciones adquiridas en la comunidad fue $15,6 \%$, similar a otros reportes en Venezuela $(16,7 \%)$ para enterobacterias con la misma procedencia ${ }^{29} y$ ligeramente superiores a lo reportado en otros países como Israel $(13,7 \%)^{36}$ e Irán $(11 \%)^{37}$. Se ha sugerido que el incremento de la proporción de microorganismos productores de BLEE en la comunidad ha sido producto de la migración desde el ambiente intrahospitalario de estos microrganismos hacia la comunidad ${ }^{38}$; $\sin ^{\text {embargo, }}$ se ha evidenciado el ingreso de bacterias productoras de BLEE desde la comunidad al hospital donde, debido a una mayor densidad de selección, se produciría un efecto de amplificación (selección) $)^{36}$.

En cuanto a la susceptibilidad in vitro de las especies aisladas en este estudio, en general se observó que los antimicrobianos con mayores tasas fueron los carbapenémicos (ertapenem e imipenem) y amikacina, todos por encima de $90,0 \%$. Esto es consistente con lo reportado en Norteamérica y Europa ${ }^{2}$ donde los agentes más activos contra los patógenos aislados fueron ertapenem $>$ imipenem $>$ amikacina $>$ piperacilina/tazobactam $>$ cefalosporinas $>$ quinolonas $>$ ampicilina/sulbactam. Sin embargo, esto difiere de lo reportado para los países de Pacífico Asiáti$\mathrm{co}^{39}$, en los que ertapenem e imipenem tuvieron tasas de actividad in vitro menores que las de amikacina $(91,7 \%)$, no obstante, su actividad fue ligeramente inferior a la encontrada en este estudio (93,9\%).

De las bacterias no entéricas, $P$. aeruginosa fue aislada en forma predominante de pacientes con infecciones nosocomiales $(70,0 \%)$ y mostró tasas de susceptibilidad muy bajas a la mayoría de los antimicrobianos evaluados (menores a 58,0\%), lo que indica que en Venezuela se ha incrementado la resistencia de esta bacteria a la mayoría de los antimicrobianos. En este estudio, todos los aislados de $A$. baumannii procedieron de infecciones adquiridas en la comunidad. Típicamente se ha descrito $A$. baumannii como un patógeno oportunista causante de infecciones nosocomiales ${ }^{40}$; sin embargo, recientemente se le ha identificado en infecciones adquiridas en la comunidad. La mayoría se ha reportado de países con clima tropical y subtropical, principalmente en pacientes con alguna co-morbilidad o asociadas con consumo excesivo de alcohol o tabaco.

Ningún aislado de $A$. baumannii fue susceptible a los antimicrobianos evaluados, a diferencia de otros reportes donde se describe sensibilidades que oscilan entre 17,9\% para ceftazidima y ciprofloxacina, hasta $35,7 \%$ para imipenem y amikacina, mostrando valores intermedios para levofloxacina, cefepime y piperacilina/tazobactam ${ }^{39}$. En Venezuela se ha identificado el gen que codifica para la carbapenemasa tipo OXA23 en aislados procedentes de varios centros de Caracas $^{41}$, la que contribuye de manera significativa en la resistencia que exhibe este patógeno a carbapenémicos ${ }^{42}$.

En general, los carbapenémicos y amikacina fueron los antimicrobianos que mejor actividad presentaron frente a todos los aislados de enterobacterias, excepto con $E$. cloacae en el que la tasa de susceptibilidad a ertapenem $(60,0 \%)$ fue la más baja exhibida por este antimicrobiano en este grupo de patógenos. A su vez, imipenem (60,0\%) tuvo comportamiento similar con E. aerogenes. En Venezuela se ha documentado la presencia de enzimas tipo KPC en E. cloacae, genotipo que se ha relacionado con la resistencia de las enterobacterias a carbapenémicos ${ }^{34}$.

En este estudio se observa un incremento de las bacterias productoras de BLEE, lo que condiciona resistencia a cefalosporinas de tercera y cuarta generación. Para cefotaxima y ceftriaxona, en este estudio se encontraron tasas de resistencia por encima de $95,0 \%$ en los patógenos productores de BLEE, ligeramente inferiores para 
ceftazidima $(80,8 \%)$ y cefepime $(73,4 \%)$, estas tasas, a su vez, fueron ligeramente inferiores a las reportadas en el Pacífico Asiático, donde se obtuvieron para el período de 2009-2010, tasas de resistencia a cefotaxima y cefepime de 98,4 y $91,5 \%$, respectivamente ${ }^{39}$ y muy superiores a las reportadas anteriormente en ocho centros en Caracas para cefepime $(17,0 \%)$ en el período $2001-2004^{33}$. Todas las cefalosporinas evaluadas tuvieron elevadas tasas de actividad in vitro para las bacterias no productoras de BLEE (92,7-98,5\%), dependiendo su actividad de la presencia de esta enzima en las enterobacterias aisladas.

En el grupo de patógenos identificados como productores de BLEE, se observó que solamente que ertapenem e imipenem tuvieron tasas de actividad superior a 98,0\% y por encima de $85,0 \%$ a amikacina, siendo estas tasas superiores a las reportadas en países del Pacífico Asiático ${ }^{39}$, donde además reportan susceptibilidad superior a $80,0 \%$ para piperacilina/tazobactam, comportamiento que no fue observado en el período estudiado $(68,1 \%)$. La actividad de imipenem se ha mantenido similar a la descrita anteriormente en Venezuela; sin embargo, la susceptibilidad a piperacilina/tazobactam ha disminuido ligeramente ${ }^{33}$. Por otro lado, los resultados en $K$. pneumonaie productoras de BLEE, con una resistencia a carbapenems de 8,3\% probablemente sea indicativo de mecanismos adicionales de resistencia.

En el grupo de bacterias sin confirmación fenotípica de producción de BLEE, se observaron tasas de susceptibilidad superior a 70,0\% para todos los antimicrobianos (no incluido ampicilina/sulbactam) excepto para imipenem, con el que se obtuvo una de las tasas más bajas para este agente, específicamente contra P. mirabilis (26,3\%), incluyendo todas las cepas aisladas en el estudio sin considerar a $P$. penneri, $P$. vulgaris y $A$. baumannii, en los que se observó $100,0 \%$ de resistencia. Esta resistencia inusual de P. mirabilis, no descrita anteriormente en Venezuela, podría estar relacionado con la degradación del antimicrobiano a través del tiempo o la concentración de Zinc en el medio, reportando falsa resistencia a imipenem, lo cual ya ha sido reportada para otras especies ${ }^{43,44}$.

Sin embargo, hay que considerar lo referido anteriormente a imipenem como menos bactericida para Proteeae que para otras enterobacterias ${ }^{45}$. Además, se ha reportado la producción de metalo- $\beta$-lactamasas $(\mathrm{M} \beta \mathrm{LM})$ por $P$. mirabilis capaz de hidrolizar a los carbapenems, exhibiendo resistencia a nuevos $\beta$-lactámicos en ambientes hospitalarios ${ }^{46} \mathrm{y}$ en infecciones adquiridas en la comunidad $^{47}$. Adicionalmente, Proteus spp. puede tener CIM elevados a imipenem por mecanismos no relacionados a la producción de carbapenemasas ${ }^{17}$.

Según reportes previos de la susceptibilidad de $E$. coli en pacientes con infección urinaria, en uno de los centros participantes de SMART (Hospital Universitario de Caracas $)^{27}$, la resistencia a cefepime, cetofaxima, ceftazidima, ceftriaxona y ciprofloxacina se ha incrementado con respecto a los obtenidos en ese estudio, todas por encima de $11,0 \%$ excepto para ciprofloxacina $(6,6 \%)$, siendo más notable este aumento en cefotaxima y ceftazidima, con diferencias de 19,1 y 15,3\%, respectivamente. Los antimicrobianos más activos para todos los aislados de E. coli fueron imipenem $(99,7 \%)$, ertapenem $(99,5 \%) \mathrm{y}$ amikacina (97,0\%). Para E. coli productora de BLEE, las tasas de resistencia en este estudio fueron muy elevadas para ciprofloxacina $(97,5 \%)$ y levofloxacina $(91,1 \%)$, ampicilina/sulbactam $(91,1 \%)$, y las cefalosporinas exceptuando cefoxitina.

En las cepas de E. coli no productoras de BLEE, los porcentajes de susceptibilidad fueron superiores a 90,0\% para todos los agentes excepto para las fluoroquinolonas y ampicilina/sulbactam. La susceptibilidad de E. coli productora de BLEE a los antimicrobianos es similar a lo reportado para esta especie en el conglomerado de países que participan en SMART, difiriendo en las tasas de susceptibilidad para piperacilina/tazobactam, ya que en este estudio fueron inferiores $(88,2 \%)$ a las del conglomerado $(99,4 \%)$. Además, las tasas de resistencia observadas en este estudio para las fluoroquinolonas son muy superiores a las observadas para ciprofloxacina $(85,4 \%)$ y levofloxacina $(84,1 \%)$ en el consolidado de SMART para 2009-2010

La actividad de la mayoría de los antimicrobianos fue ligeramente inferior frente al total de aislados de K. pneumoniae en comparación con E. coli, excepto para ciprofloxacina y levofloxacina $(70,0 \%$, cada una), ampicilina/ sulbactam $(52,0 \%)$, en las que las susceptibilidades fueron superiores. Las tasas de susceptibilidad más elevadas de $K$. pneumoniae productora de BLEE fue para ertapenem, imipenem y cefoxitina ( $91,7 \%$, cada una). A diferencia de la actividad de amikacina y piperacilina/tazobactam frente a $E$. coli y $P$. mirabilis, las tasas de actividad de estos antimicrobianos en $K$. pneumoniae fueron la más bajas exhibida por ellos frente a las bacterias aisladas en este estudio, excepto con K. oxytoca, A. baumannii y $P$. aeruginosa. Ampicilina/sulbactam no tuvo actividad frente a $K$. pneumoniae productora de BLEE, siendo esto igual a lo reportado en Europa e inferior que lo observado en Norteamérica $(33,3 \%)^{2}$.

En general, las tasas más bajas de actividad obtenidas en el período estudiado, al margen de ampicilina/sulbactam, fueron ciprofloxacina $(58,3,0 \%)$ y levofloxacina $(60,0 \%)$, con tasas menores en los bacilos gramnegativos no fermentadores $P$. aeruginosa $(42,9 \%)$ y A. baumannii $(0 \%)$ para ambos fármacos, diferente a lo descrito para ciprofloxacina en Norteamérica ${ }^{49}$. Debido a la elevada tasa de resistencia de las bacterias entéricas y no entéricas a las fluoroquinolonas, no sería adecuado recomendar estos antimicrobianos como terapia inicial en las ITU. Aunque los datos in vitro no siempre equivalen a los 
resultados clínicos, especialmente en las ITU donde las fluoroquinolonas a menudo alcanzan altas concentraciones, las tasas de resistencia descritas para Latinoamérica (38,7\%), Asia (33,2\%); Europa (29,4\%); Pacífico del Sur (25,5\%); África (25\%); Norteamérica $(23,5 \%)$ y Medio Oriente $(20,7 \%)$, sugieren que las alternativas a la terapia con fluoroquinolonas pueden merecer consideración en entornos de creciente resistencia a estos antimicrobianos ${ }^{50}$. En este sentido, se ha sugerido el uso de nitrofurantoína como terapia inicial ${ }^{9,25,26,51}$, pero no en aquellos casos con infección importante del tracto urinario superior ni cuando hay compromiso sistémico ${ }^{49}$.

En conclusión, E. coli es el patógeno responsable en las ITU adquiridas en la comunidad y de origen nosocomial, seguida de K. pneumoniae. La proporción de cepas productoras de BLEE en las infecciones nosocomiales fueron más elevadas que las obtenidas de las infecciones originadas en la comunidad. En total, la proporción de BLEE se ha incrementado ligeramente para E. coli y $K$. pneumoniae, y continúa en baja proporción para $P$. mirabilis. Los antimicrobianos con mejor actividad contra las bacterias gramnegativas aisladas fueron los carbapenémicos y amikacina, inclusive en las cepas productoras de BLEE. Para el total de aislados, las tasas de susceptibilidad fueron superiores a $80,0 \%$ en la mitad de los antimicrobianos evaluados con excepción de cefotaxima, ceftazidima, ceftriaxona, ciprofloxacina, levofloxacina y ampicilina/sulbactam, teniendo estos tres últimos las tasas más elevadas de resistencia, incluso más elevadas que las reportadas previamente. Las tasas de susceptibilidad a los doce antimicrobiano evaluados en el presente estudio sugieren una necesaria revisión de los esquemas terapéuticos para el tratamiento empírico de la ITU en Venezuela, con la finalidad de evitar la disemina- ción de mecanismos de resistencia bacteriana y limitar el uso de los fármacos que en la actualidad se disponen para el tratamiento de la ITU en este país.

\section{Resumen}

Introducción: La resistencia antimicrobiana de patógenos causantes de infecciones del tracto urinario (ITU) es un problema creciente, que complica su tratamiento efectivo. La vigilancia es necesaria para orientar la terapia empírica apropiada. Objetivo: Documentar los patrones de susceptibilidad de bacterias gramnegativas aisladas de pacientes con ITU a doce antimicrobianos como parte del Estudio de Monitoreo de Patrones de Resistencia en Venezuela (SMART). Materiales y Métodos: Entre 2009-2012, un total de 472 bacterias gramnegativas fueron aisladas de pacientes hospitalizados con ITU. Los aislados fueron enviados a un Laboratorio Central (Central Laboratory of International Health Management Associates) para confirmar su identificación y realizar pruebas de susceptibilidad según recomendaciones del Instituto de Estándares Clínicos y de Laboratorio. Resultados: Enterobacteriacea comprendió 96,6\% del total, donde Escherichia coli (76,9\%) y Klebsiela pneumoniae $(10,6 \%)$ fueron las más frecuentes. En $21,6 \%$ de los aislados fueron detectadas $\beta$-lactamasas de espectro extendido (BLEE). Los antimicrobianos con mejor actividad fueron ertapenem, imipenem y amikacina $(>90,0 \%)$; ligeramente menor para amikacina $(85,1 \%)$ en cepas productoras de BLEE. Las tasas de resistencia a quinolonas y ampicilina/ sulbactam fueron elevadas (40 y 64\%, respectivamente). Conclusiones: Estos datos sugieren una necesaria revisión de los esquemas terapéuticos para el tratamiento empírico de las ITU en Venezuela.

\section{Referencias bibliográficas}

1.- Najar M, Saldanha C, Banday K. Approach to urinary tract infection. Indian J Nephrol 2009; 19 (4): 129-39.

2.- Hoban D, Lascols C, Nicolle L, Badal R, Bouchillon S, Hackell M, et al. Antimicrobial susceptibility of Enterobacteriaceae, including molecular characterization of extended spectrum betalactamase-producing species, in urinary tract isolates from hospitalized patients in North America and Europe: result from the SMART study 2009-2010. Diagn Microbiol Infect Dis 2012; 74: 62-7.

3.- Wagenlehner F, Cek M, Naber K, Kiyota H, Bjerklund-Johansen T. Epidemiology, treatment and prevention of healthcare-associated urinary tract infections. World J Urol 2012; 30: 59-67.

4.- Foxman B. Epidemiology of urinary tract infection: Incidence, morbidity, and economic cost. Am J Med 2002; 113 Supl 1A: 5-13.

5.- Kucheria R, Dasgupta S, Sacks S, Khan M, Sheerin N. Urinary tract infections: new insights into a common problem. Postgrad Med J 2005; 81: 83-6.

6.- Hooton T, Scholes D, Hughes J, Winter C, Roberts P, Stapleton A, et al. Prospective study of risk factors for symptomatic urinary tract infection in young women. N Engl J Med 1996; 335: 468-74.

7.- Foxman B. Recurring urinary tract infection: incidence, morbidity, and economic costs. Am J Public Health 1990; 80 (3): 331-3.

8.- Scholes D, Hooton T, Roberts P, Stapleton A, Gupta K, Stamm W. Risk factors for recurrent urinary tract infection in young women. J Infect Dis 2000; 182: 1177-82.

9.- Gupta K, Hooton T, Naber K, Wullt B, Colgan R, Miller L, et al. International clinical practice guidelines for the treatment of acute uncomplicated cystitis and pyelonephritis in women: A 2010 update by the Infectious Diseases Society of America and the European Society for Microbiology and Infectious Diseases. Clin Infect Dis 2011; 52: e103.

10.- Alós J. Capítulo 1: Epidemiología y etiología de la infección urinaria comunitaria. Sensibilidad antimicrobiana de los principales patógenos y significado clínico de la resistencia. Carlos Pigrau, editor. Infecciones Urinarias. Madrid: Salvat; 2013, p. 1-11.

11.- Kashef N, Esmaeeli G, Shahbazi S. Antimicrobial susceptibility patterns of community acquired uropathogens in Tehran, Iran. J Infect Dev Ctries 2010; 4 (4): 202-6.

12.- Grundmann H, Klugman K, Walsh T, Ramon-Pardo P, Betuel S, Khan W, et al. A framework for global surveillance of antibiotic resistance. Drug Resist Updat 2011; 14: 79-87. 
13.- Hawser S. Surveillance Programmes and Antibiotic Resistance: Worldwide and Regional monitoring of antibiotic resistance trends. Handb Exp Pharmacol 2012; 211: 31-43.

14.- Alós J. Epidemiología y etiología de la infección urinaria comunitaria. Sensibilidad antimicrobiana de los principales patógenos y significado clínico de la resistencia. Enferm Infecc Microbiol Clin 2005; 23 Supl 4: 3-8.

15.- Grundmann H, O’Brien T, Stelling J. Chapter 2: Surveillance to track antimicrobial use and resistance. World Health Organization (WHO), editor. The evolving threat of antimicrobial resistance. Options for action. Geneva: WHO Press; 2012. p. 12-30.

16.- Morrissey I, Hackel M, Badal R, Bouchillon S, Hawser S, Biedenbach D. A review of ten years of the Study for Monitoring Antimicrobial Resistance Trends (SMART) from 2002 to 2011. Pharmaceuticals 2013, 6: 1335-46.

17.- Clinical and Laboratory Standards Institute (CLSI). Performance Standards for Antimicrobial Disk Susceptibility Tests, Approved Standard-11th ed. CLSI document M02-A11. Wayne, PA: The Institute; 2012.

18.- Myoung H. Univariate analysis and normality test using SAS, STATA, and SPSS. Univariate analysis and normality test: 1 . Technical Working Paper; 2008. Disponible en:www.indiana. edu/ statmath/stat/all/normality/normality.pdf. (accedido el 01 de octubre de 2012].

19.- Norman G, Streiner D. Bioestadística. Madrid, España: Mosby/Doyma Libros, S.A.; 1996.

20.- Armitage P, Berry G. Estadística para la Investigación Biomédica, $3^{\text {a }}$ ed. Madrid: Hartcourt Brace; 1997.

21.- Ministerio del Poder Popular para la Salud (MPPS). Anuario de Morbilidad 2009. Caracas: MPPS; 2010.

22.- Ministerio del Poder Popular para la Salud (MPPS). Anuario de Morbilidad 2010. Caracas: MPPS; 2011.

23.- Ministerio del Poder Popular para la Salud (MPPS). Anuario de Morbilidad 2011. Caracas: MPPS; 2012.

24.- Peleg A, Hooper D. Hospital-acquired Infections due to gram-negative bacteria. N Engl J Med 2010; 362: 1804-13.

25.- Khawcharoenporn T, Vasoo S, Ward E, Singh $K$. High rates of quinolone resistance among urinary tract infections in the ED. Am J Emerg Med 2012; 30 (1): 68-74.

26.- Mohammad-Jafari H, Jafar M, Nemate I, Saffar $\mathrm{H}$, Khalilian A. Increasing antibiotic resistance among uropathogens isolated during years 20062009: Impact on the empirical management. Int Braz J Urol 2012; 38: 25-32.

27.- Villarroel E, Navarro P, Ramos R, Andrade E, Bolívar A, Marcano J. Escherichia coli identificadas en pacientes con infecciones urinarias: Sensibilidad antimicrobiana. Rev Soc
Ven Microbiol 2002; 22 (1): 18-21.

28.- Requena I, De Pace C, Torres P, Padrón A. Resistencia antibiótica de bacterias causantes de infección del tracto urinario. Saber 2007; 19 (2): 150-8.

29.- Guevara A, Machado S, Manrique E. Infecciones urinarias adquiridas en la comunidad: epidemiología, resistencia a los antimicrobianos y opciones terapéuticas. Kasmera 2011; 38 (2): 87-97.

30.- Ronald A. The etiology of urinary tract infection: Traditional and emerging pathogens. Dis Mon 2003; 49: 71-82.

31.- Jacoby G, Muñoz-Price S. The new $\beta$-lactamases. N Engl J Med 2005; 352: 380-91.

32.- Pitout J. Infections with extended-spectrum $\beta$-lactamase producing Enterobacteriaceae. Changing epidemiology and drug treatment choices. Drugs 2010; 70 (3): 313-33.

33.- Torres L, Gagliotta V, Torres O, Benítez M, Domínguez M, Pedroza R. $\beta$-Lactamasas de espectro expandido en enterobacterias aisladas en Centros de Salud de Caracas. Rev Soc Ven Microbiol 2006; 26 (2): 80-8.

34.- Marcano D, De Jesús A, Hernández L, Torres L. Frecuencia de enzimas asociadas a sensibilidad disminuida a betalactámicos en aislados de enterobacterias, Caracas, Venezuela. Rev Panam Salud Publica 2011; 30 (6): 529-34.

35.- Perozo-Mena A, Castellano-González M, Ginestre-Pérez M, Harris B. Caracterización molecular y detección de betalactamasas de espectro extendido en cepas de $E$. coli y $K$. pneumoniae aisladas en las unidades de cuidados intensivos de un hospital universitario. Kasmera 2007; 35 (2): 91-106.

36.- Ben-Ami R, Schwaber M, Navon-Venezia S, Schwartz D, Giladi M, Chmelnitsky I, et al. Influx of extended-spectrum beta-lactamaseproducing Enterobacteriaceae into the hospital. Clin Infect Dis 2006; 42: 925-34.

37.- Syeda N, Lolith L, Manju S. A study of prevalence of extended-spectrum $\beta$-lactamase producing Enterobacteriaceae from urinary isolates in community setting. Insight Bacteriology 2013; 2 (2): 10-4.

38.- Cantón R, Valverde A, Novais A, Baquero F, Coque T. Evolución y panorama actual de las BLEE. Enferm Infecc Microbiol Clin 2007; Supl 2: 2-10.

39.- Po-Liang L, Yung-Ching L, Han-Siong T, Yu-Lin L, Yuag-Meng L, Cheng-Mao H, et al. Epidemiology and antimicrobial susceptibility profiles of Gram-negative bacteria causing urinary tract infections in the Asia-Pacific region: 2009-2010 results from the Study for Monitoring Antimicrobial Resistance Trends (SMART). Int J Antimicrob Agents 2012; 40 Suppl 1: 37-43.

40.- Pedroza R, Cuotto W, Velázquez O, Torres L, Rodríguez V. Caracterización de plásmidos de Acinetobacter baumannii en tres centros hospitalarios. Rev Fac Med (Caracas) 2002; 25 (1): 80-2.

41.- Cuaical N, Delgado Y, Anzola Y, Marcano D, Torres L. Detección de carbapenemasas tipo OXA en aislados de Acinetobacter baumannii de diferentes centros hospitalarios de Caracas, Venezuela. Rev Soc Ven Microbiol 2012; 32: 95-100.

42.- Pinzón J, Mantilla J, Valenzuela E, Fernández F, Álvarez C, Osorio E. Caracterización molecular de aislamientos de Acinetobacter baumannii provenientes de la unidad de quemados de un hospital de tercer nivel de Bogotá. Infectio 2006; 10 (2): 71-8.

43.- White R, Kays M, Friedrich L, Brown E, Koonce J. Pseudoresistance of Pseudomonas aeruginosa resulting from degradation of imipenem in an automated susceptibility testing system with predried panels. J Clin Microbiol 1991; 29 (2): 398-400.

44.- Tsakris A, Pantazi A, Pournaras S, Maniatis A, Polyzou A, Sofianou D. Pseudo-outbreak of imipenem-resistant Acinetobacter baumannii resulting from false susceptibility testing by a rapid automated system. J Clin Microbiol 2000; 38 (9): 3505-7.

45.- Yourassowsky E, Van der Linden M, Crokaert F. Antibacterial effect of meropenem and imipenem on Proteus mirabilis. J Antimicrob Chemother 1990; 26: 185-92.

46.- Miriagou V, Papagiannititsis C, Tzelepi E. Detecting VIM-1 production in Proteus mirabilis by an imipenem-dipicolinic acid double disk synergy test. J Clin Microbiol 2010; 48 (2): 667-8.

47.- Tsakris A, Ikonomidis A, Poulou A, Spanakis N, Pournaras S, Markou F. Transmission in the community of clonal Proteus mirabilis carrying VIM-1 metallo- $\beta$-lactamase. J Antimicrob Chemother 2007; 60: 136-9.

48.- Hoban D, Nicolle L, Hawser S, Bouchillon S, Badal R. Antimicrobial susceptibility of global inpatient urinary tract isolates of Escherichia coli: results from the Study for Monitoring Antimicrobial Resistance Trends (SMART) program: 2009-2010. Diagn Microbiol Infect Dis 2011 ; 70: 507-11.

49.- Vásquez Y, Hand W. Antibiotic susceptibility patterns of community-acquired urinary tract isolates from female patients on the US (Texas)México border. JAR 2004; 4 (2): 321-6.

50.- Bouchillon S, Hoban D, Badal R, Hawser S. Fluoroquinolone resistance among Gram negative urinary tract pathogens: global SMART program results, 2009-2010. Open Microbiol J 2012; 6: 74-8.

51.- Páez A, Rojas J, Guiaquirian L, Danna N. Manejo de la infección urinaria no complicada. Borregales L, Giordano F, Contreras L, editores. Primer Consenso Venezolano de Infecciones Urinarias 2011. Sociedad Venezolana de Urología. Caracas: ATEPROCA 2011; p. 41-52. 